DOI 10.17816/transsyst201844112-128

(C) E. Chao ${ }^{1}$, J. Venturi ${ }^{2}$.

${ }^{1}$ University of Pennsylvania, Department of Electrical and Systems Engineering (Philadelphia, USA)

${ }^{2}$ Rethink Studio

(New York, USA)

\title{
OPERATIONAL BREAKDOWN AND PERFORMANCE MEASURE OF THE TRANSCONTINENTAL HIGH-SPEED MAGLEV
}

Background: Magnetic Levitation (Maglev) systems have a noticeable operating track record in about a dozen countries. Higher speed maglev technology has been built for many intercity and regional lines in China, Germany, Japan, South Korea, United States, Brazil, and other countries. Maglev developers claim that the transcontinental high speed system can outperform the existing HSR and air transport and can achieve higher speed, have lower energy consumption and life cycle costs, attract more passengers, and boost regional economy. The article presents a systematic breakdown of the proposed transcontinental high speed Maglev system and pinpoints critical operational components and implementation measures. The analyses reach the following discussions on the three most important system characteristics.

Firstly, the transcontinental high speed Maglev had to make trade-offs among passenger access time to total travel time, station density to daily maximum operating speed, and operating strategy to daily skip-stop, express, as well as other accelerated services.

Secondly, the correlation between systems capacity management and vehicle interior space design (e.g. seats) has a serious impact on operators' long-term financial condition. The involvement of identifying the equilibrium between these two factors in a linear algebra method is substantial.

Thirdly, the transcontinental high speed Maglev station must serve as the multimodal transportation hub. To attract passengers; accordingly, increase the ridership and farebox recovery, an unified transfer service on schedule coordination has to be incorporated into the system. Timed Transfer Systems (TTS) had the proven capability of increasing service reliability across different modes. Based on these discussions, the framework and direction of transcontinental high speed Maglev strategic planning is becoming sensible.

Aim: The article addresses the major system design elements of transportation planning and pinpoints corresponding operational strategies, which are useful for the planning and design of maglev. The study will assist system designers, network planners, and operators to understand where the technical and operational boundaries are for this particular mode. Knowing the boundary is useful for the design, planning, and operations of the system.

Methods: The efforts of literature reviews focus on two fields: composition of major system design elements and interrelation with other modes of transportation. The method examines the foundation of maglev planning.

Results: First, the benefit of speed increase cannot be hasty generalized. The assessment of speed increase needs to break down to different beneficiaries (e.g. operator, passenger, and the community). Second, system capacity depends on its operating speed, service frequency, load factor, and vehicle size. These four factors further determine the operational feasibility of 
the maglev. Finally, in a dispersed travel pattern, TTS increases transfer reliability and unifies different lines of headway to improve service reliability.

Conclusion: Certain cities and countries are facing similar transportation issues. They are trying to learn from each other. The efforts focus on the establishment of efficient transit systems and the dedicated action to adopt a new mode of transportation (e.g. maglev) for intracity, intercity, transcontinental commutes. The article offers tangible values on transportation planning, systems design, and operation performance, which are critical for the development of the maglev system.

Keywords: Transcontinental Maglev, Strategic Planning and Implementation Measures, Systems Design, Operation Performance

\section{INTRODUCTION}

The feasibility and application of low, medium, and high-speed maglev to intracity, intercity, transcontinental commute are receiving more and more attention. For example, Changsha Maglev Express is the first low-medium maglev line in China with an average speed of $65 \mathrm{~km} / \mathrm{hr}$ [1]. Virgin Hyperloop One, Hyperloop Transportation Technology, and Hardt Hyperloop are dedicated to developing transcontinental high-speed operational prototypes for both passenger and freight [2-4]. While these firms are trying to commercialize the high-speed maglev system, many interesting challenges lie in front of it. Invalid transportation planning concepts spread out on the internet by claiming that the system will be an on-demand service without a fixed schedule [5], which failed to consider the interrelations between station density, area coverage, and passenger access time as well as total travel time. At a technical level, the new system is particularly sensitive to station density, area coverage, vehicle size, vehicle capacity, throughputs, and many other operational elements while pursuing the maximum speed.

\section{AIM}

The article addresses the common system design elements of transportation planning and pinpoints corresponding operational strategies. The study will assist system designers, network planning, and operators to understand where the technical and operational boundaries are for this particular mode. Knowing the boundary is useful for the design, planning, and operations of the system.

\section{ANALYSES}

No matter for the intracity (low and medium speed) or intercity or transcontinental (high speed) maglev system, many transportation planning trade- 
offs have to be considered. The first trade-off refers to passenger access time and travel time. The purpose of establishing a station is to provide a convenient access to the passenger, but too many stations would increase travel time due to the corresponding halts. Thus, evaluating the conflict of station density and speed could assess whether the advantages of speed increase outweigh the disadvantages. Second, system capacity depends on its operating speed, service frequency, load factor, and vehicle size. Examining these four factors would clarify its operational feasibility. Finally, the computation of TTS schedule and its pros and cons are given.

\subsection{System design}

Passenger Access and Travel Time. The travel time of passengers on transit line passenger time (PT) consists of two main concepts: access to/from the stations, including waiting for a train $\mathrm{PT}_{a}$ and travel time on the line $\mathrm{PT}_{t}$ as shown in Fig. 1 [6].

$$
\mathrm{PT}=\mathrm{PT}_{a}+\mathrm{PT}_{t}
$$

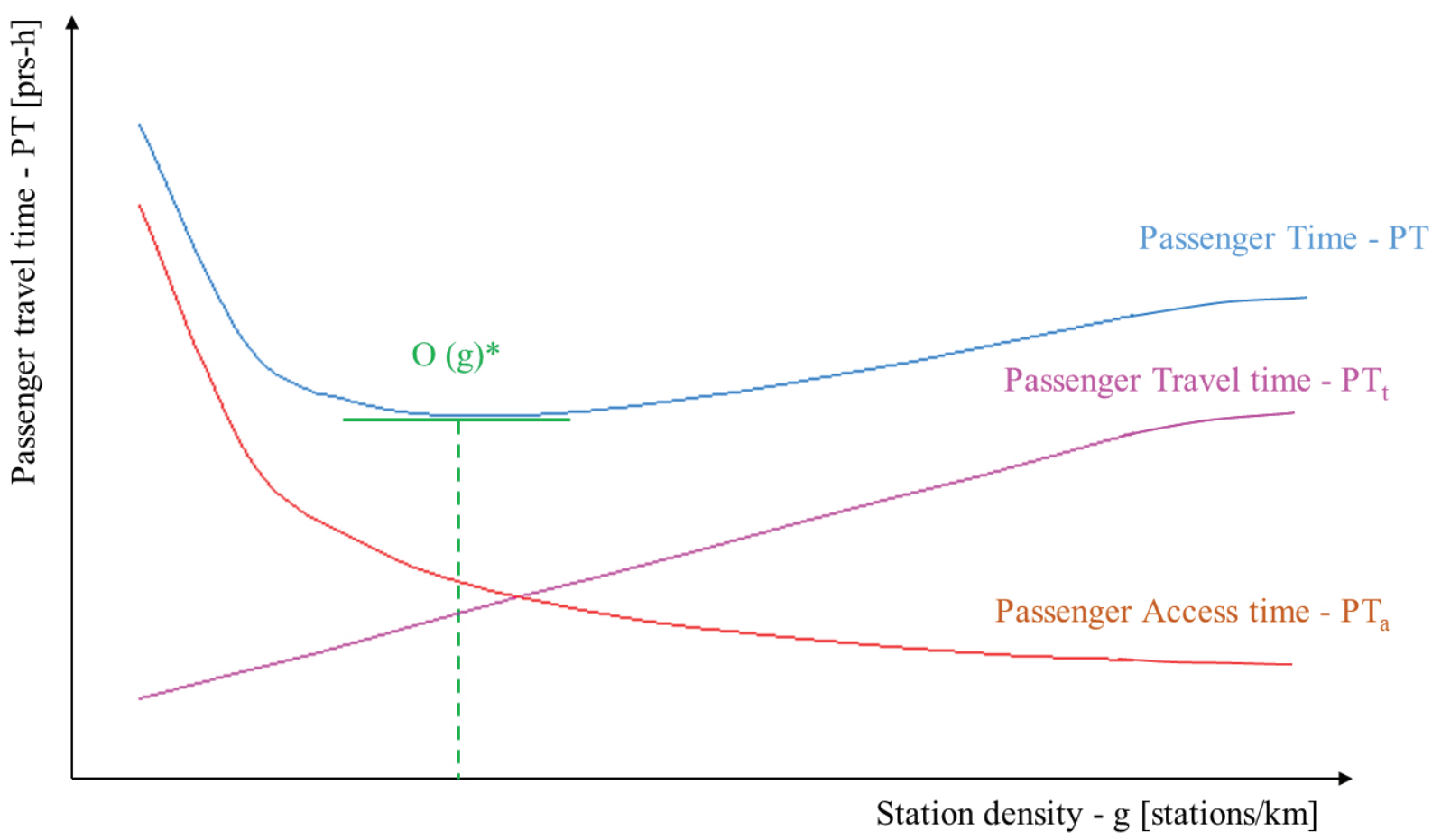

Fig. 1. Passenger travel time as a function of station density [6] 
The number and locations of stations along a line influence both access and travel time. The number of stations on a line with a given distance is often analyzed through the station density - g, defined as station per $\mathrm{km}$, or the inverse of the average station spacing. For example, if the average spacing is $0.8 \mathrm{~km}$, station density is $g=1 / 0.8=1.25$ station $/ \mathrm{km}$.

Considering passenger travel times, station density must be based on the optimum value trade-off between access $\left(\mathrm{PT}_{a}\right)$ and travel times $\left(\mathrm{PT}_{t}\right)$.

1. The increase of station density $-\mathrm{g}$ results in a decrease of average distance and access time to station $\mathrm{PT}_{a}$

2. The growing of station density $-\mathrm{g}$ on the line results in the increase of passenger travel time $-\mathrm{PT}_{t}$

When station spacing becomes too short that trains cannot reach their maximum speed, the additional delay happens so that the marginal increase in travel time begins to decline. The total passenger travel time curve PT shows an optimal station density $-\mathrm{O}(g)^{*}$. If passenger distribution along a line is uniform, the optimal station density is determined by the trade-off. If passenger distribution is non-uniform, such distribution influences the optimal station locations and results in variable spacing [6-7].

Planning of stations faces a basic dilemma: closer stations (short spacing in between) result in better area coverage and easier access for a larger number of potential passengers. However, short station spacings cause lower operating speed and possibly larger vehicle size, as well as higher construction and station maintenance costs. Longer station spacings result in the opposite situation: high speed and better operation, but with a line passing through areas without serving them, since there are no stations. A portion of potential passengers is then lost [8].

Station Density vs. Speed. Transit operation has a sensitive relationship between the number of stations and speed or disutility. As shown in Fig. 2, when station density g [st's $/ \mathrm{km}$ ] is low (say, only two terminal stations for a single line), travel speed can go very fast, but the total disutility is also very high, mainly due to lots of unserved areas (resulting in low ridership). As the station density increases, speed will become lower, then more passenger will be served although the overall travel time increases. This also results in a lower cost per passenger.

Fig. 3 shows distribution of Q stations in a single line (from the left) and speed (from the right). Transit operators mostly operate trains under the lower disutility scenario, the distribution between stations and speed will be at the equilibrium point $E$ : $\mathrm{q}_{\mathrm{g}}$, the number of station, and $\mathrm{q}_{\mathrm{vc}}$, the operating speed. This situation can be seen in the real world where there is a high-speed maglev with an average speed of $350 \mathrm{~km} / \mathrm{hr}$ and a high-speed rail (HSR) line with an average speed of $250 \mathrm{~km} / \mathrm{hr}$. If one could make an impact on the other, for example, $\Delta \mathrm{q}$ 


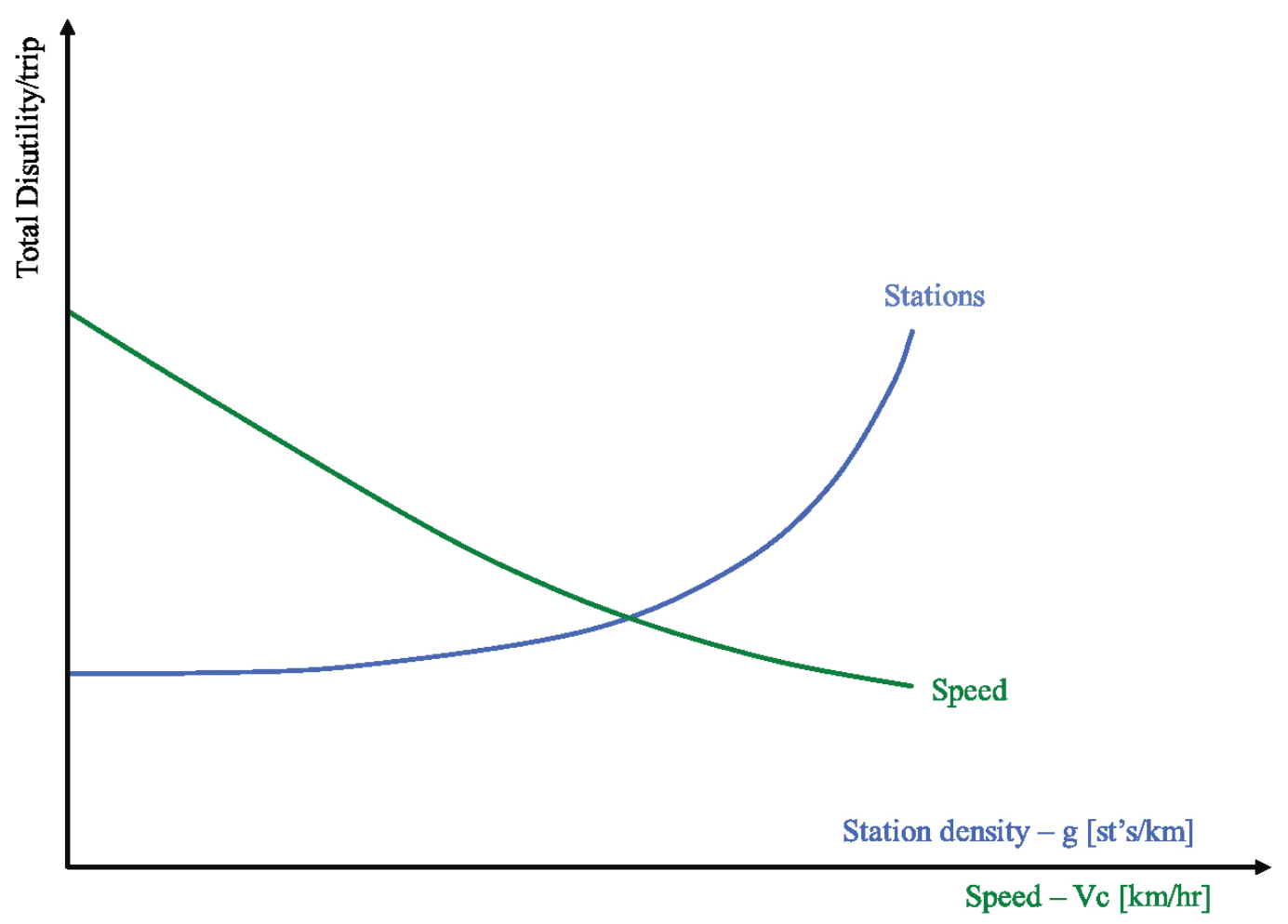

Disutility: user cost, travel time, unserved areas, system externalities

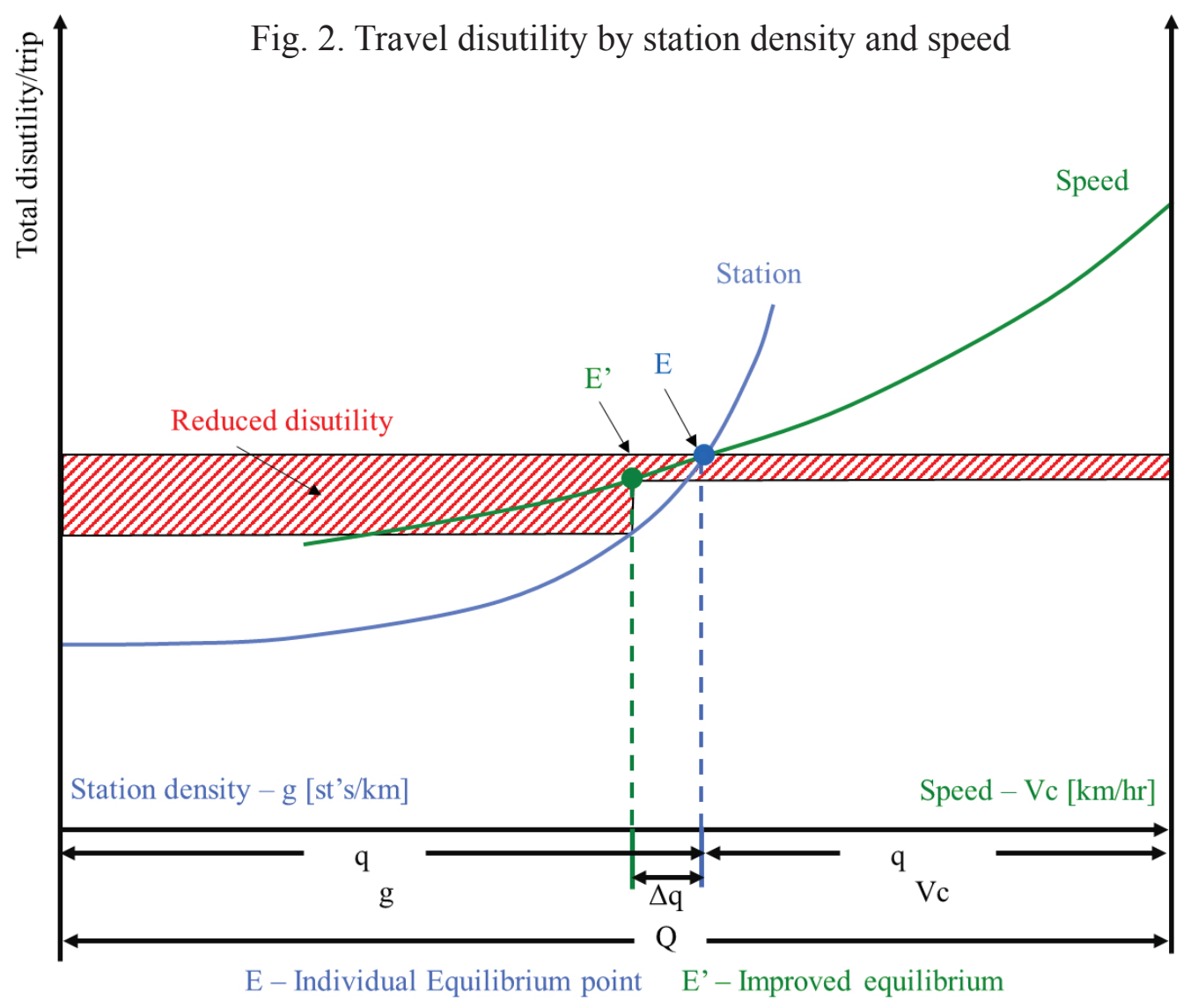

Fig. 3. Travel distribution between station density and speed 
shows the number of travelers deciding to travel on a single line when the number of stations decreases, its operating speed can be increased. Therefore, the travelers on the line would benefit, so identifying station density and speed leads a system optimum - minimum total disutility.

Maglev system plans to challenge the existing modes of transportation or create a new mode to massively shorten the citywide commuting time. One must understand this relationship between station density and speed, and they apply two sets of design principle or operating strategy to plan for a "flexible" maglev. The term "flexible" in transportation systems planning always comes with trade-offs [9]. As abovementioned, the trade-offs are between passenger access time and passenger travel time as well as station density and speed. Thus, if pursuing the maximum speed of this new mode of transportation, one should understand the importance of the two sets of interchangeable design principles:

- Method I: Increase speed;

- Method II: Decrease station density.

Fig. 4 shows how these two sets of measures result in a shift of the equilibrium point from the individual equilibrium point (IE) toward the system

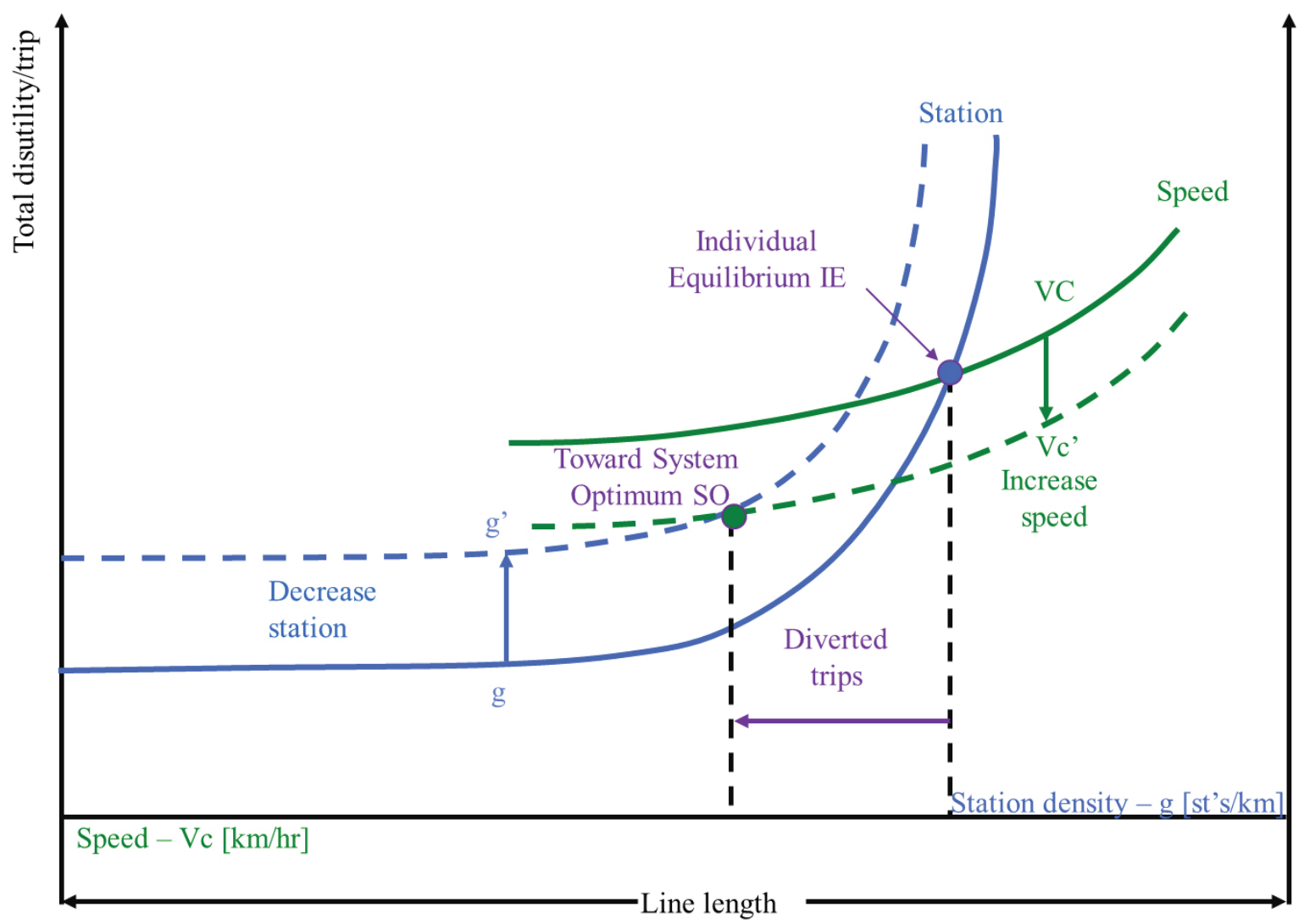

Fig. 4. Operation strategy for shifting individual equilibrium to system optimum 
optimum (SO): to increase speed move the $\mathrm{V}_{\mathrm{C}}$ curve down to $\mathrm{V}_{\mathrm{C}}$ ', whereas station density moves the g curve up to g'. The result is a shift of operating more stations to fewer stations so that trains can travel at a higher speed, known as skip-stop and express services, this goes from the initial IE toward the SO by the individual decisions of travelers and operator, and it remains stable there. The diagram shows that the total disutility of travel in both modes, which was initially at the IE level, and has been reduced to SO.

The corresponding operation measure of station spacing on any section along a line should be a function of the ratio of the number of passengers with origins and destinations along the ridership distribution vs. the number passengers on the trains passing though the same section preferring to skip the stop due to time loss. The greater of this ratio, the more the station be established. On the other hand, where the volume of through passengers dominates the volume of local passengers, station spacing should be long. In the extreme case, if there is a section without any passengers waiting to board and alight, there should be no stations. An important consequence of this relations is that, with respect to passenger time (PT), uniform/ fixed station spacing is seldom optimal. Overall, station density $-\mathrm{g}$ varies with the distribution of passenger demand along the line [6-8].

After addressing the interrelation and trade-off of passenger time, station density, and speed, cost of stations is worth to be discussed.

Cost of stations. Each station involves certain investment cost and operating cost for station operation and maintenance, stopping trains, and for larger transit unit due to longer cycle time. For equal local conditions, the incremental cost per station is constant when station are far apart, but it decreases slightly when spacings become so short that trains cannot reach maximum speed because the incremental time and energy consumption per stopping are then slightly reduced. Thus, the total cost of stations increases with their density, but at a decreasing rate, as plotted in Fig. 5 [6].

In a nutshell, identifying the optimal station density by overlapped multiple operating factors is able to find the optimal value for station density as Fig. 6. shown. Among all these factors, passenger time is the most decisive factor and has a strong correlation with station density.

\subsection{System capacity}

Optimal Vehicle Size \& Capacity. To analyze how maglev system could outperform other transit modes under which conditions, it is critical to understand 


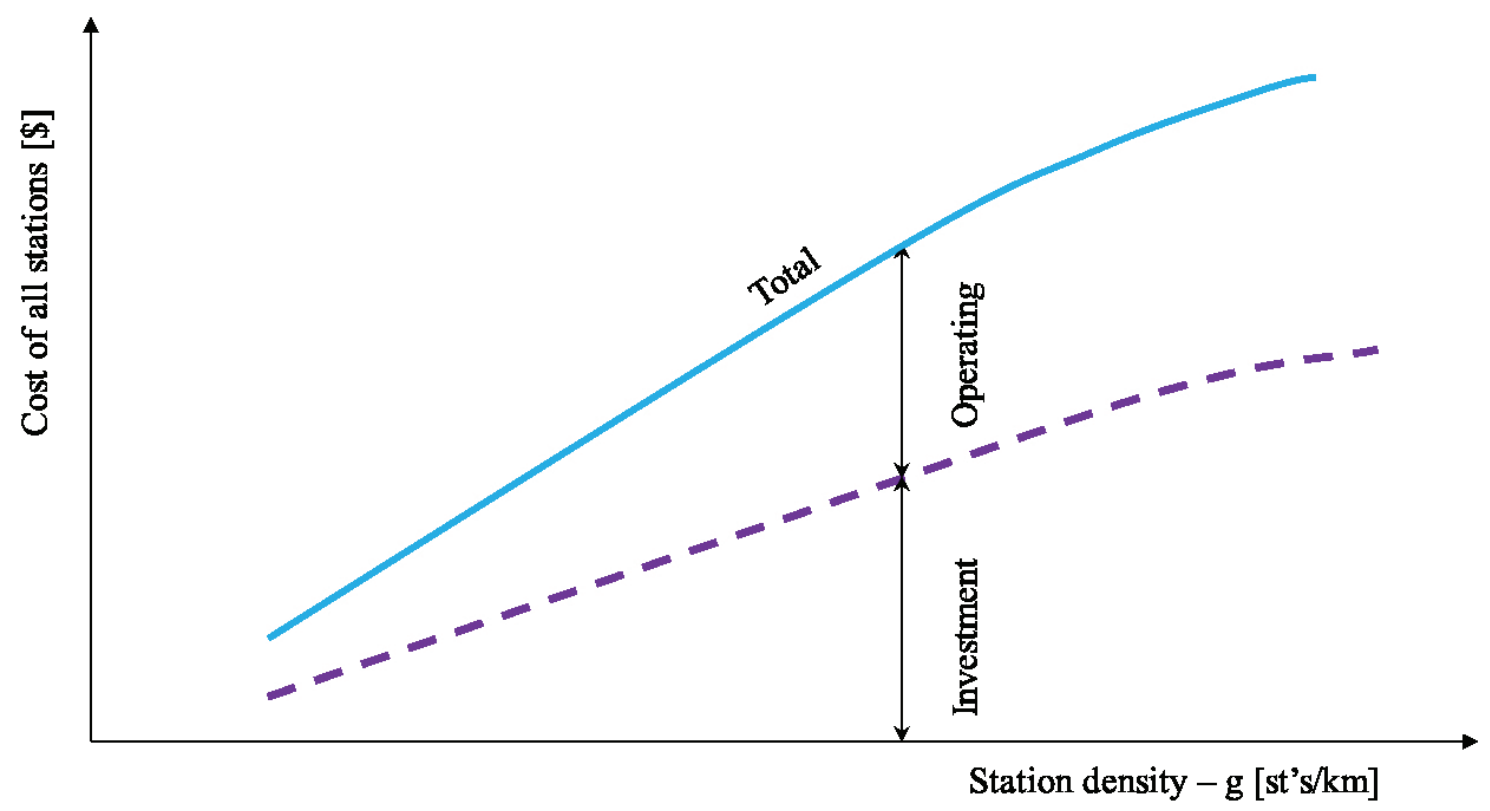

Fig. 5. Total cost (investment and operating) of stations by density on a fixed line [6]

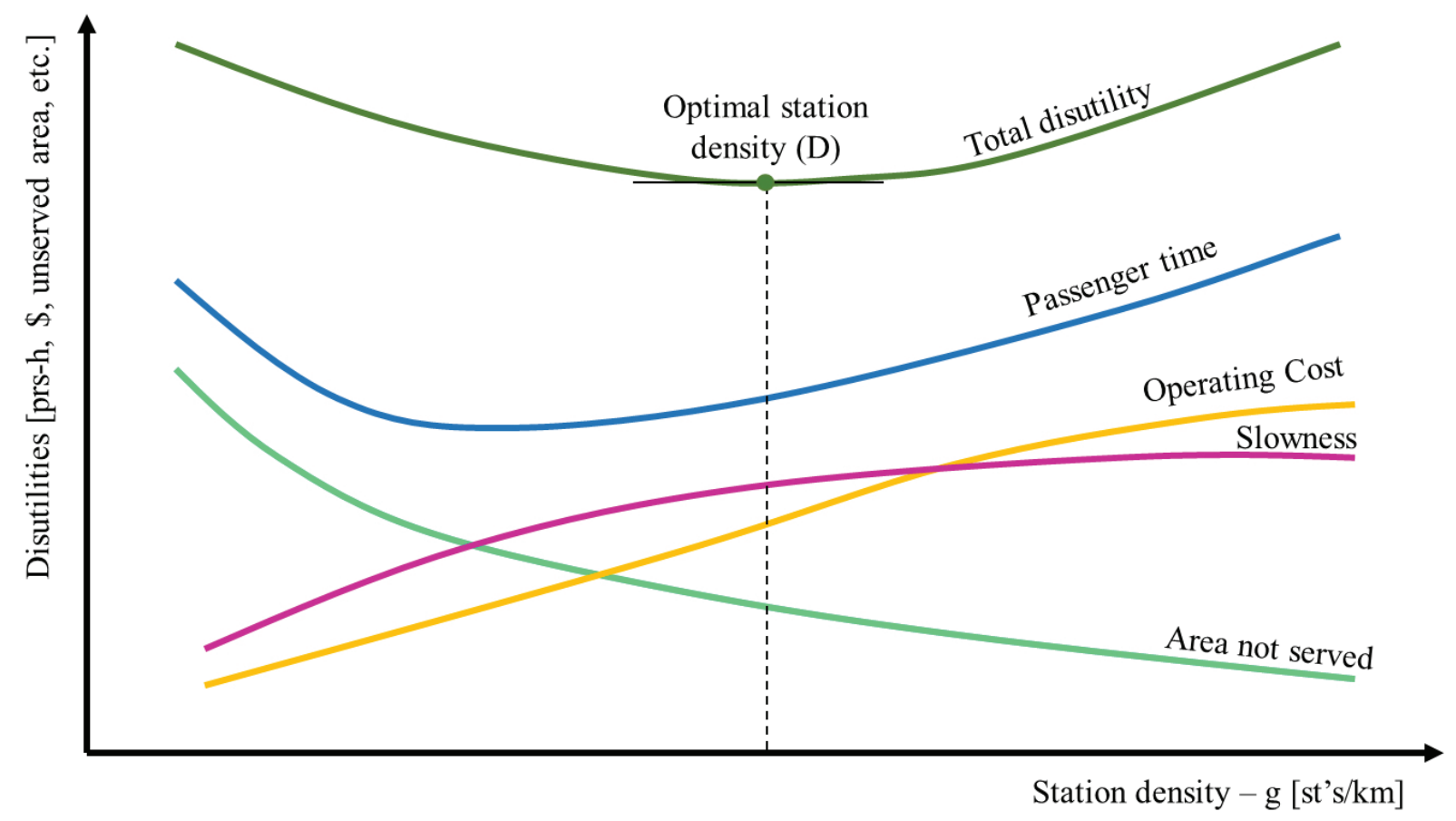

Fig. 6. Optimal station density on network planning [6] 
the system capacity and operation boundaries. One of the common methods to distinguish modes is the vehicle size associated with system capacity.

Fig. 7 shows the relations among service headway, transit unit (TU) capacity, and the line capacity. To challenge the existing modes of transportation, one must firstly define the operating boundaries with a holistic consideration of vehicle capacity and size of transport units. The critical condition for which every system must be designed is the peak hour, precisely, the maximum passenger volume expanded to hourly volume. From an operation perspective, it would be financially difficult to justify the exclusive right-of-way (ROW) and the investment of a fully automated system if its design capacity were less than $2000 \mathrm{sps} / \mathrm{hr}$ or actual passenger volume between 1500 to $1800 \mathrm{prs} / \mathrm{hr}$. For example, at Point A1 and A2, the system is able to ship $25 \times 50$ and $10 \times 100$ [frequency $\times$ space] units respectively per hour. However, the operating cost and passenger/cargo per unit cost is high compared to Point B (3000 psr/hr) and Point C (7200 prs/hr). Therefore, the maglev system capacity is very volatile to TU capacity, ideally greater than 50 spaces for passengers TUs [10].

The operation domain is the limit of the purple-dashed box. The left boundary is limited due to TU capacity, that is, the offered spaces. The right

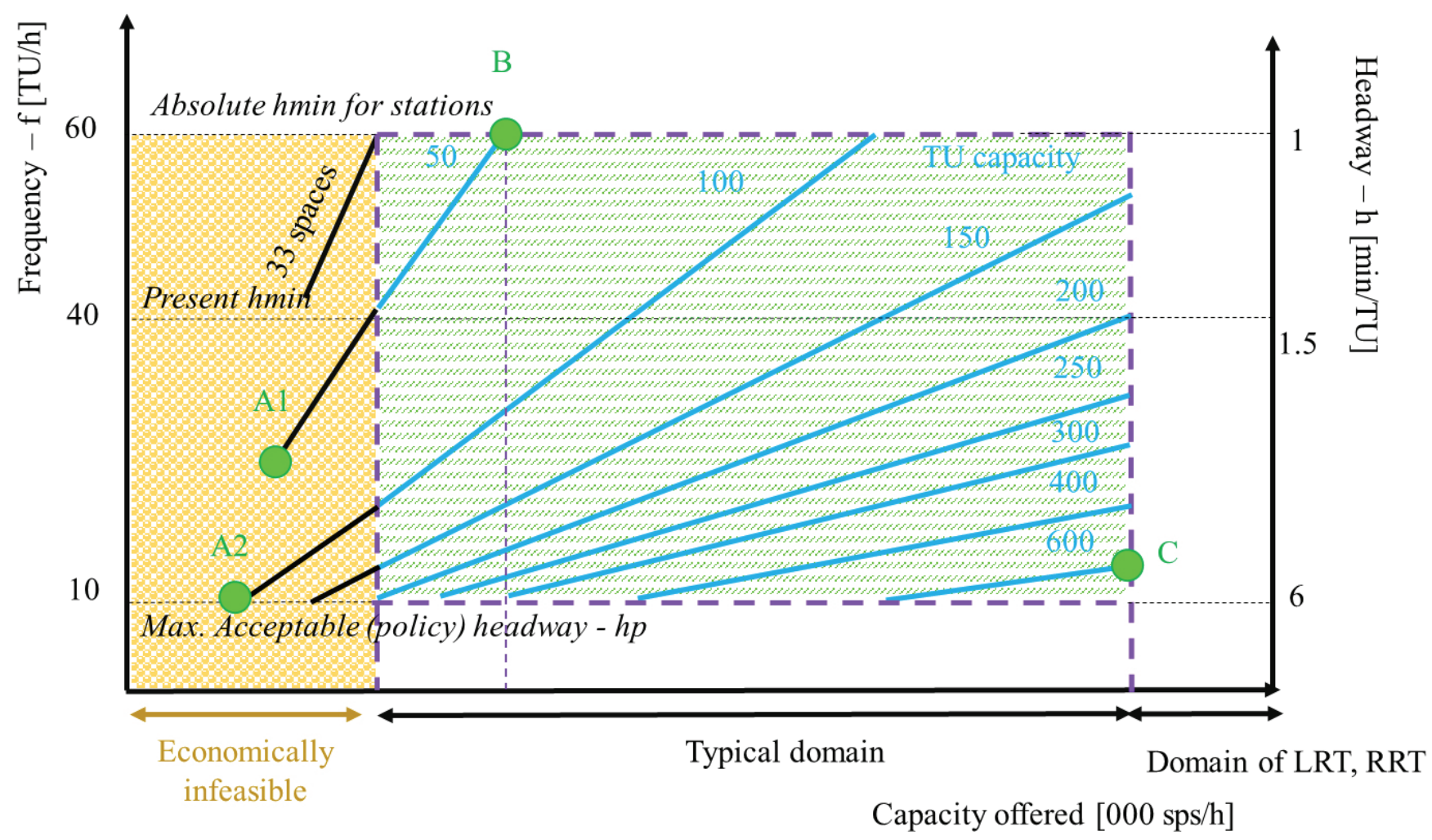

Fig. 7. Transit unit capacity by frequency and line capacity [10] 


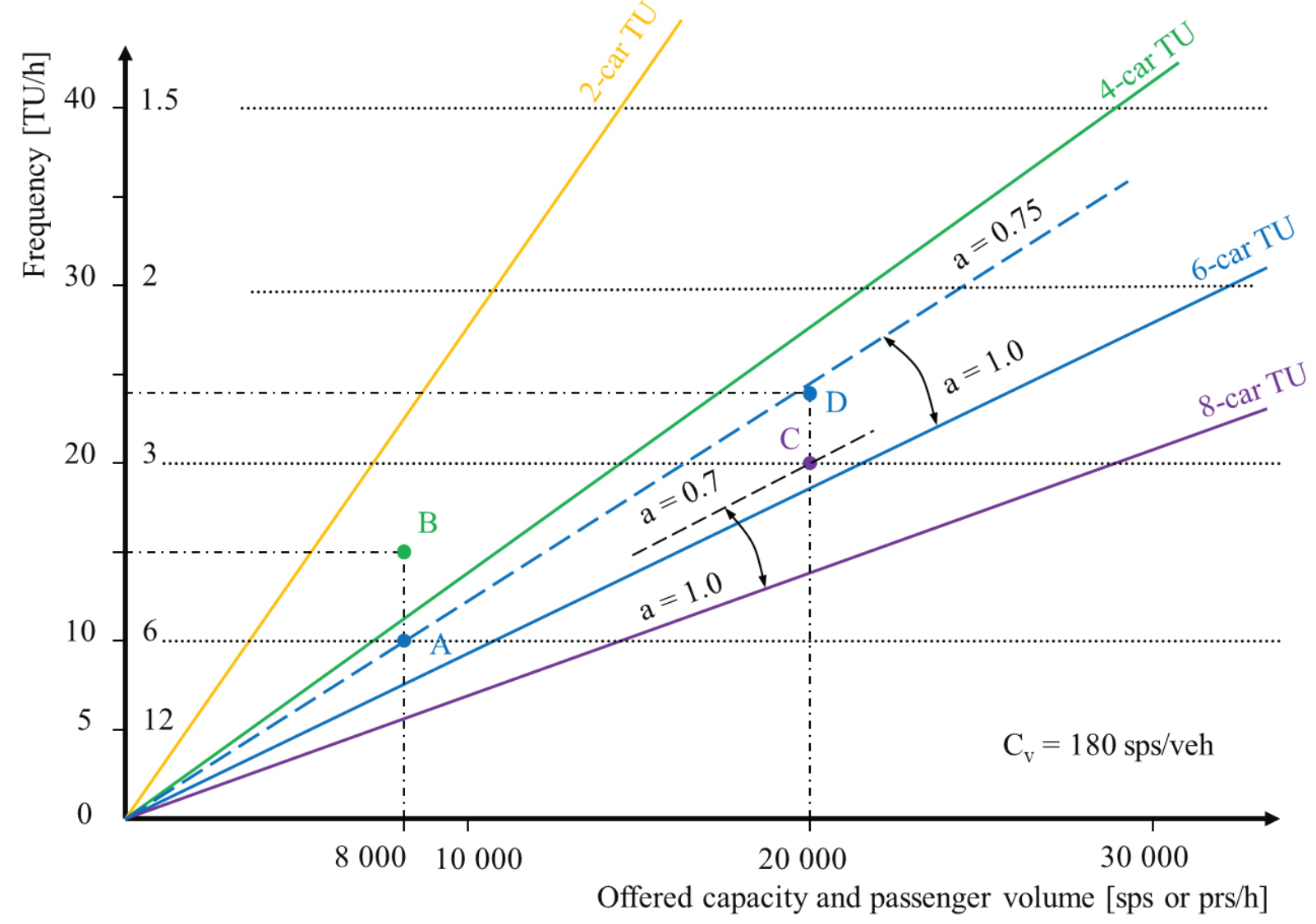

Fig. 8. Operation strategy: transit unit capacity and headway on schedule design [6]

and bottom boundaries are limited due to its maximum TU capacity with the consideration of headway simultaneously. The fact is that large vehicles cannot run short headway considering the length of the vehicle and safety distance. Around the globe, Moscow and Barcelona metros have had experience with operating vehicles within $75 \mathrm{sec}(48 \mathrm{TU} / \mathrm{hr})$ of headway with a 6-car train and $90 \mathrm{sec}(40 \mathrm{TU} / \mathrm{hr})$ headway with a 5-car train respectively under Automated Train Control (ATC) [11-12].

Assuming the maglev TU capacity were smaller than these two metros' both TU length and capacity will able to operate its fleets with $60 \mathrm{sec}$ headway $(60 \mathrm{TU} /$ $\mathrm{hr}$ ), then the upper boundary were to be established. Such an approach has been commonly used in linear algebra. Considering all the above, a typical operating domain falls into the green area.

\section{Guidance of TU Size, Frequency \& Load}

Fig. 8. can be used to select the optimal combination of transit unit (TU) size $-\mathrm{n}$, operating speed $-C_{v}$, service frequency $-f$, headway $-\mathrm{h}$, and load factor $-\alpha$ for any scheduling period of the day. 
The diagram is based on the equation:

$$
C=n \times C_{v} \times \alpha \times f
$$

The equation shows four different trains consisting of: TU sizes of 2, 4, 6,8-cars, and the line capacity with operations at different frequency/headway and load factor $\alpha$. Each slope line shows the values for a given TU size at full occupancy, $\alpha=1$. A blue-dashed line shows the capacities offered by six-car TUs with $\alpha=0.75$.

Assumed offered capacity is 180 seats per vehicle. During mid-day period $\mathrm{P}_{\max }-8,000 \mathrm{prs} / \mathrm{hr}$, reasonable choices would be to operate 6-car TUs at $\mathrm{h}=6 \mathrm{~min}$ with $\alpha=0.75$ - point A on the diagram; or 4-car TUs at $\mathrm{h}=4$ min with $\alpha=0.75$ point B. Suppose that the peak period has $\mathrm{P}_{\max }=22000 \mathrm{prs} / \mathrm{hr}$; then the choices may be to operate 8 -car TUs at $\mathrm{h}=3$ min and $\alpha=0.70$ - point $\mathrm{C}$, or 6 -car TUs at 2.5 min and $\alpha=0.77$ - point $\mathrm{D}[6]$.

After examined the major system design elements of transportation planning, it is time to analyze the interdependent operation strategies across different modes. That is, how could one system work cooperatively with another to create a cohesive schedule to increase overall passenger gain and system ridership.

\subsection{System unification}

Timed Transfer System (TTS) is a network consisting of several transit lines and one or more transit centers at which transit units from all intersecting lines arrive simultaneously, allowing passenger transfers in all directions. TTS coordinates different lines' schedule and commonly used in medium-sized cities and suburban areas to fulfill the seamless integration and connectivity. Without the application of TTS, passenger transfers among lines involve a higher «resistance», because a transfer may cause delay and require passenger re-orientation and extra walking between mezzanines and platforms on different lines [6, 13-14].

\section{TTS SCHEDULING}

- In an uncoordinated scheduling system (Fig. 9a.), the basic operating elements (length, cycle time, fleet size, operating speed, and number of vehicles or transport unit on a route) for each route are independent in the network.

- In TTS network the headway (and therefore frequency) must be the same on all routes (Fig. 9b.), or an exact multiple of headways on other route, only with 


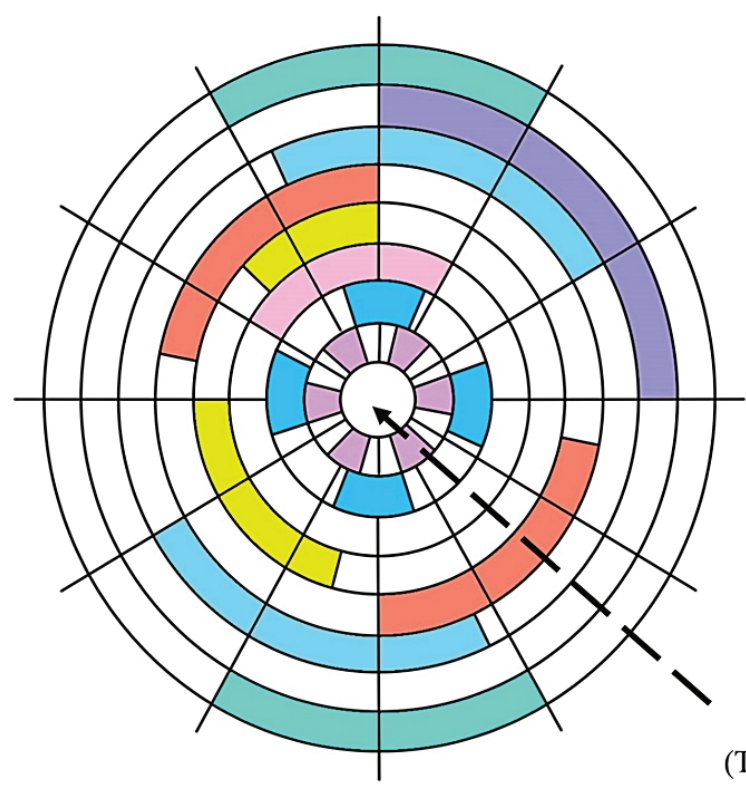

a. Independent line schedules

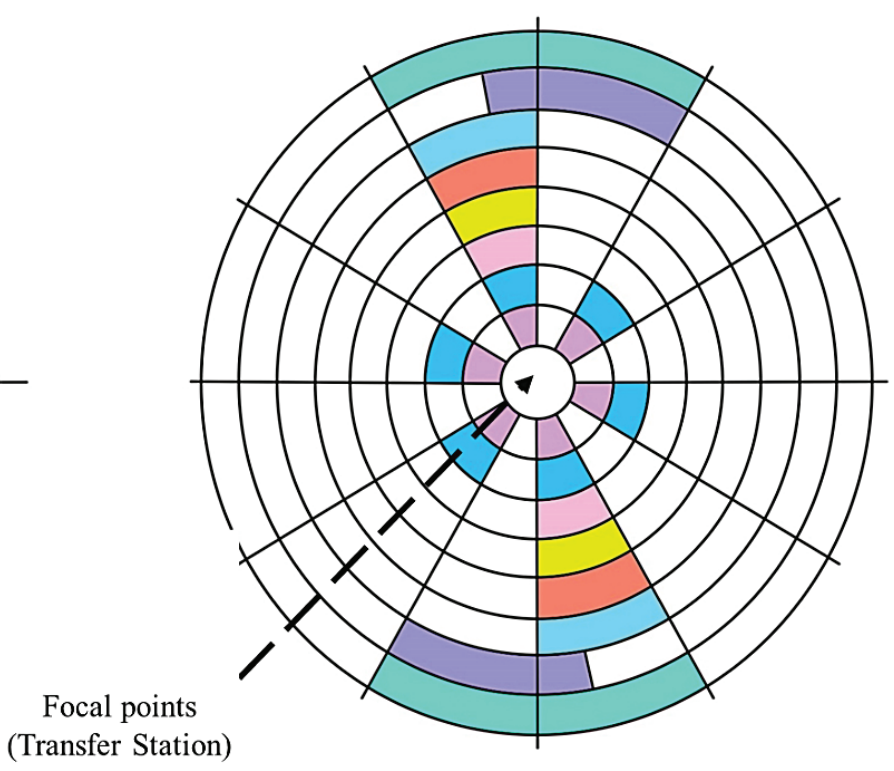

b. TTS schedules

Fig. 9. Clock-type diagram showing two types of line schedules at a transit center [13]

few exceptions for special purpose. For example, all routes coming to a focal point or transfer station must have headways of 5,10,15 min, or exceptionally, 30 or $60 \mathrm{~min}$. Headways are required to be divisible into one to enable easy memorization of schedules by passengers.

- The value of very short pulse headway $(h)$ can be used only on high reliable systems with a fully controlled Right-of-Way A. As a Virgin Hyperloop One video shown, the systems have simultaneous arrivals of vehicle or transport unit from different lines and exchange of passenger (every 5, or even every 2.5 mins) [14]. However, such short headways could increase operation and maintenance cost. Another aspect should be considered while planning the headway is the demand, level of services, and expected reliability of service on each route.

- For every case, each line must have the same headway. That is, the same ratio of cycle time and number of transport units on the line $[6,13]$ :

$$
h=\frac{T 1}{N 1}=\frac{T 2}{N 2}=\ldots \ldots
$$




\section{TTS PROS AND CONS}

TTS execution relies on the same ratio of cycle time and number of TUs on the line. Compared with conventional operation, TTS has the following advantages $(+)$ and disadvantages $(-)$ :

+ Transfers among all routes meeting at each transit center are much faster and more convenient;

+ Transit service represents a multidirectional, unified network serving a variety of trips, as opposed to services restricted to individual routes in networks without convenient transfers;

+ Large, distinct terminals provide a much greater number of services than smaller ones for individual routes;

+ Due to the better services (network, schedules, public image, terminals), TTS attracts a substantially larger patronage and plays a more important role than conventional networks;

- TTS generally requires a higher investment (terminals, information) and operating costs (more vehicles on routes), which may or may not be offset by higher revenues;

- Some passengers may have less direct routing and additional delays during layovers of through routes at transit centers;

- TTS is more vulnerable to delays: missed expected connections cause considerable aggravation of passengers;

- "Pulsing" at terminals may cause congestion on access routes and it requires large terminal capacities [13].

\section{CONCLUSION}

The diversity among cities and countries in terms of their historic, geographic, social, and strategic positions dictate to the requirements for a variety of approaches and solutions to urban transportation problems. Policies and solutions cannot be directly transferred from one to another; however, many fundamental problems are similar, and the exchange of experience can be useful in resolving the sophisticated problems faced by cities.

Many global cities are either in the transition stage of rethinking cities' longterm competitiveness or in the development stage of large-scale city and regional planning. Common facts have been found in these two settings: the outstanding commitments on the selection and establishment of efficient transit systems and the 
dedicated action to adopt a new mode of transportation (e.g. maglev) for intracity, intercity, transcontinental commutes.

While cities are trying to learn from each other, a dedicated effort to understand systems design and operational measurement is indispensable. Transit system, by nature, has its own boundary. The boundary needs to be meticulously studied. Fig. 10 shows an evaluation of speed increase to different beneficiaries. In addition, vehicle size has a strong correlation with system capacity and maximum load. Knowing system's capacity and operation limitations are useful to examine whether one system could outperform another under which condition. Fig. 11 shows whether the maglev system could outperform the existing modes depend on speed, cost, and ridership. The condition is given under the same line capacity so that the operating speed of medium-speed maglev is higher than light rail in a right-of-way $\mathrm{B}$ environment and high-speed maglev is operating faster than HSR in a right-of-way A environment.

The emerging of a new mode is to complement the existing one, rather than compete with. After all, different modes have different functions. Therefore, TTS is an implementation tool to coordinate different lines of headway to increase system reliability and seamless transfers. TTS improves service cohesiveness where travel

Event

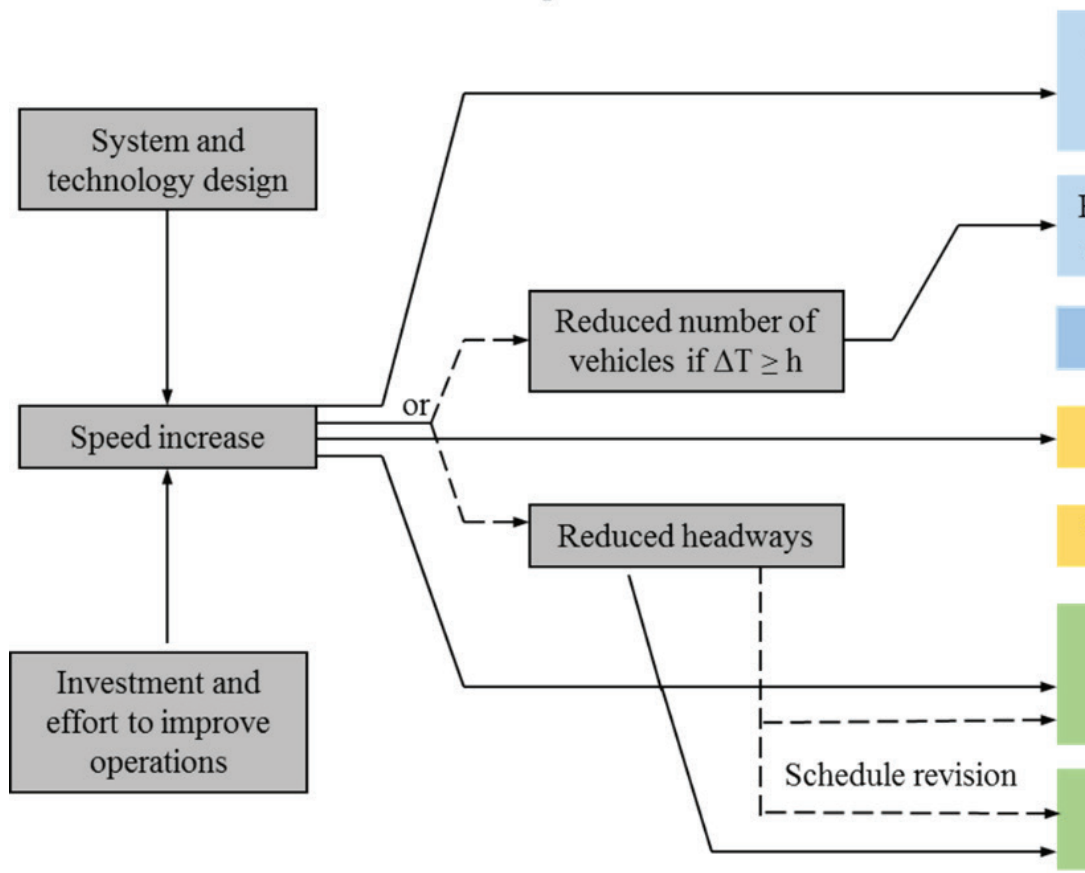

Reduced congestion -increased mobility
Benefits

Reduced operating costs (street transit modes)

Reduced investment and operating costs

Increased revenue

Travel time saved

Waiting time saved

New passengers (diverted from automobile)

New passengers (induced trips)

Existing passengers

New passengers

Community

Fig. 10. Evaluation of speed increase [10] 


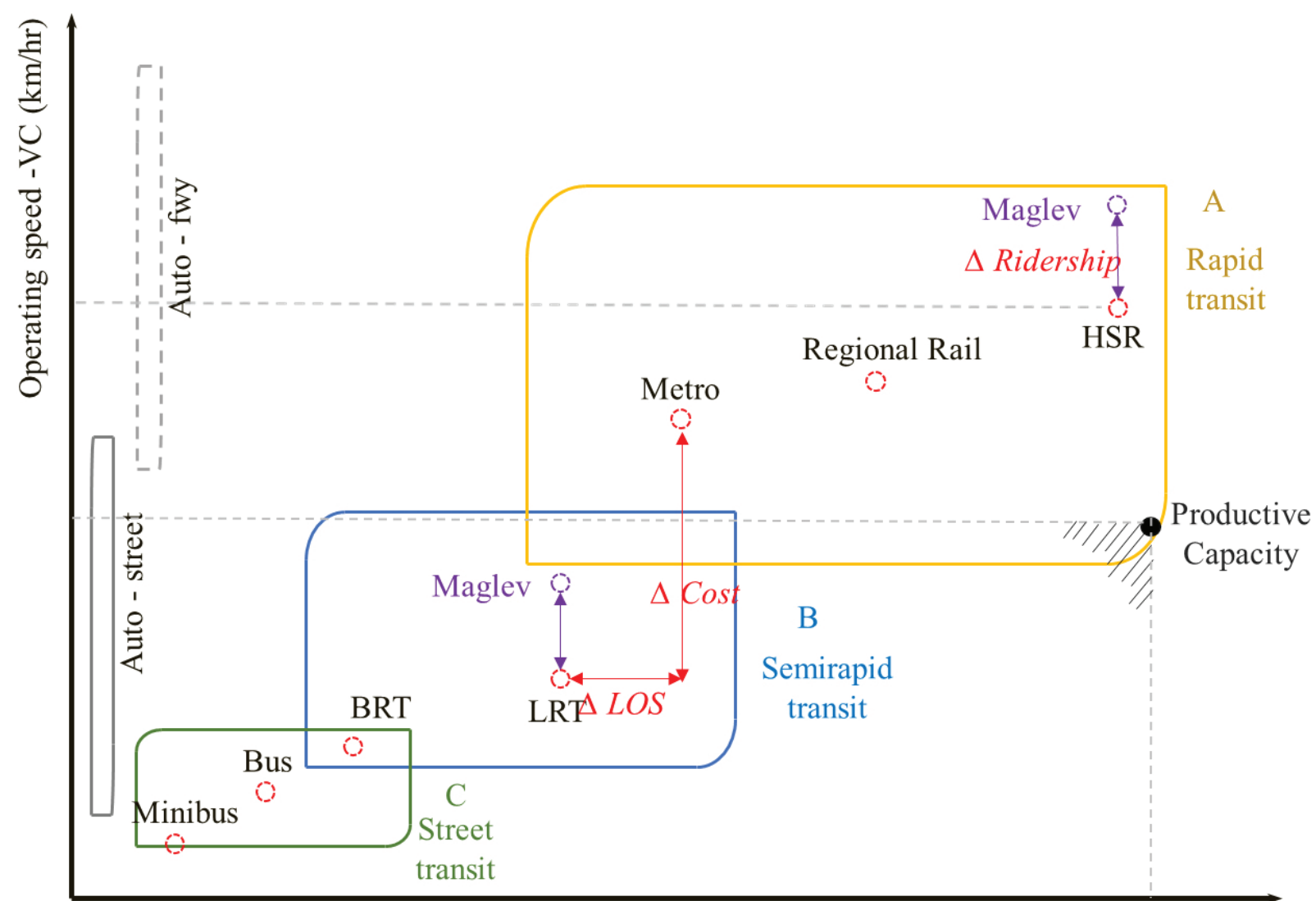

Line capacity $-\mathrm{C}($ spaces $/ \mathrm{hr} \times 1000)$

Fig. 11. Line capacity, operating speed, and productivity capacity by modes [6]

demand is characterized by dispersed O-D patterns (many-to-many) and demand density is rather uniform and major trip generators are located at several locations dispersed throughout the areas, with moderate concentrations at each one. This results in the demand for transit routes with rather similar headways converging on several different nodes.

While developing a new mode of transportation no matter for intracity, intercity, or transcontinental, certain efforts need to be carried: reducing the total disutility and avoiding mutually conflicting policies to achieve an intermodal balanced transportation system. A plan always comes with a purpose. A broad vision of the city-transportation relationships and the creation of unified services are interdependent with counties' long-term economic outlook [15-17]. 


\section{References}

1. Changsha Maglev Express [Internet]; 2017 [cited 2018 May 12] Available from: http:// enghunan.gov.cn/SP/ChangshaMaglevTrain/.

2. Virgin Hyperloop One [Internet]; c2017-2018 [updated 2018]. Available at: https:// hyperloop-one.com/.

3. Hyperloop Transportation Technology [Internet]; c2017-2018 [updated 2018]. Available at: http://www.hyperloop.global/.

4. Hardt Hyperloop [Internet]; c2017-2018 [updated 2018]. Available at: https://hardt.global/ research.

5. Lloyd R. A New Cargo Brand Built For An On-Demand World [Internet]. Los Angeles: Rob Lloyd. 2018 Apr [cited 2018 May 12]. Available at: https://hyperloop-one.com/blog/ new-cargo-brand-built-demand-world.

6. Vuchic VR. Urban Transit: Operations, Planning, and Economics. Hoboken, NJ: Wiley \& Sons. 2005.

7. Vuchic VR. Rapid Transit Interstation Spacings for Maximum Number of Passengers. Transportation Science. 1969;3(3):214-232. doi: 10.1287/trsc.3.3.214

8. Vuchic VR, Newell GF. Rapid Transit Interstation Spacings for Minimum Travel Time. Transportation Science. 1968;2(4):303-339. doi: 10.1287/trsc.2.4.303

9. Vuchic VR. Concept of Flexibility in Transportation System Analysis. High Speed Ground Transportation Journal. 1971;5(1):53-61.

10. Vuchic VR. Urban Public Transportation Systems and Technology. Englewood Cliffs, NJ: Prentice-Hall; 1981.

11. Moscow Mayor Official Website. Moscow Metro to launch fourth automated train [Internet]. Moscow; 2017 [cited 2018 May 12]. Available from: https://www.mos.ru/en/ news/item/20965073/.

12. TMB. Barcelona's driverless line 9/10 automated metro project [Internet]. Spain; 2018 [citied 2018 May 12]. Available from: https://www.tmb.cat/en/about-tmb/transportnetwork-improvements/automated-metro/which-lines.

13. Vuchic VR, et al. Timed Transfer System Planning, Design and Operation. Report DOTI-83-28 to UMTA, DOT. Washington; 1983 Oct.

14. The Hyperloop One System - UAE [video]; Virgin Hyperloop One Nov 8, 2016 Available at: https://www.youtube.com/watch?v=fze5spdN3nU. Accessed: November 8, 2016.

15. Vuchic VR. Livable Cities - Will Transportation Professionals Answer the Challenge. Transportation Research News. 2003:22-26.

16. Vuchic VR. Transportation for Livable Cities. New Brunswick, NJ: CUPR, Rutgers University. 2010.

17. Gehl J. Cities for People. Washington, D.C.: Island Press; 2010. 
Information about the authors:

Eugene (Wubin) Chao, Technical Assistant for Dr. Vukan R, Vuchic, University of Pennsylvania, Department of Electrical and Systems Engineering, Towne Building 220 South 33rd Street, Philadelphia PA 19104-6315, U.S.A

E-mail: chawu@alumni.upenn.edu

\section{Jim Venturi;}

E-mail:jim.venturi@rethinkstudio.org

\section{To cite this article:}

Chao E, Venturi J. Operational Breakdown and Performance Measure of the Transcontinental High-Speed Maglev. Transportation Systems and Technology. 2018;4(4):112-128. doi: 10.17816/ transsyst201844112-128 